\title{
Generalised coherent states and Bogoliubov transformations
}

\author{
R F Bishop and A Vourdas \\ Theoretical Physics Group, Department of Mathematics, University of Manchester Institute \\ of Science and Technology, PO Box 88, Manchester M60 1QD, UK
}

Received 8 July 1985, in final form 16 December 1985

\begin{abstract}
We study the properties of the states $U_{2}(\rho, \theta, \lambda)|A\rangle$ where $U_{2}$ is an operator associated with the group $S U(1,1)$, and $|A\rangle$ is a standard (atomic or Glauber) coherent state defined in terms of the usual boson creation and destruction operators $a^{\dagger}$ and $a$. We show how these states may be viewed as ordinary coherent states in terms of the Bogoliubov quasiparticles whose creation and destruction operators $b^{\dagger}$ and $b$ are associated with the operators $a^{\dagger}$ and $a$ by a Bogoliubov transformation. As an important example of the use of these states, we show that they are the coherent states associated with a uniformly accelerated (Rindler) observer moving through Minkowski space. Our previous results then simply show how the Minkowski (inertial) vacuum appears to the Rindler observer as a black-body radiator with a Planckian distribution corresponding to a temperature proportional to the proper acceleration.
\end{abstract}

\section{Introduction}

Since the introduction into quantum mechanics of the by now very well known standard (atomic or Glauber (1963)) coherent states, more general coherent states which are associated with particular irreducible representations of various Lie groups have also been introduced by various authors (Barut and Girardello 1971, Radcliffe 1971, Perelomov 1972, 1975, 1977, Feshbach and Tikochinsky 1977). Furthermore, other coherent states not necessarily of this type have also been specially constructed with reference to particular problems or applications (e.g. Nieto and Simmons 1979).

The physical motivation that leads us in the present paper to extend the range of application of the standard coherent states is our desire to develop a rather broad framework in which to embed the general phenomenon of clustering within a manybody medium. In the first instance we restrict ourselves to pairing correlations, but we have clearly in mind extensions to correlation phenomena concerning bound clusters of more than two particles. Since much of condensed matter physics depends ultimately on such correlations, any further attempt to motivate or to justify qualitatively our results seems superfluous at this stage. Their ultimate justification must clearly rest on their applicability and their power to suggest generalisations.

The standard coherent states are conventionally defined with respect to a set of boson creation and destruction operators $a^{\dagger}$ and $a$ respectively, as the eigenstates of the destruction operator $a$. Continuing for the purposes of initial discussion with a system of identical bosons, the introduction of the concept of correlated pairs leads us to consider the pairing operators $a^{\dagger 2}, a^{2}$ and $a^{\dagger} a$. In $\S 2$ we show how, since these operators provide a simple realisation of the Lie algebra $\mathrm{SU}(1,1)$, they can be used to construct a set of generalised coherent states associated with the corresponding Lie 
group SU $(1,1)$. We also show that these generalised coherent states may very usefully be viewed as eigenstates, not of the annihilation operator $a$, but rather of some new destruction operator $b$ which can be associated with the operators $a$ and $a^{\dagger}$ via a Bogoliubov transformation. Furthermore we show that, although the new coherent states are indeed standard coherent states with respect to the new operators $b$ and $b^{\dagger}$, they have many very interesting and useful properties in connection with the operators $a$ and $a^{\dagger}$ which have not previously been studied.

We should mention that other authors (Barut and Girardello 1971, Perelomov 1975, 1977, Feshbach and Tikochinsky 1977) have also previously introduced and studied other coherent states of the group $\operatorname{SU}(1,1)$. However, we stress firstly that the states discussed here are different from those previously considered; and secondly, and perhaps more importantly, that whereas the previous works have provided hints as to the relationship of their generalised coherent states with the Bogoliubov transformation, in the present paper this relationship is demonstrated very clearly and explicitly. In particular we show how merely by starting with the concept of pairing and hence from the pairing operators given explicitly above, we are led inevitably via the general concepts of coherent states to the Bogoliubov transformation itself. A further philosophical contrast with previous work is that we regard this particular way of viewing the Bogoliubov transformation as being very important and quite central to our stated aims. Thus, by contrast with almost all other discussions of pairing phenomena in which the Bogoliubov transformation is introduced in an extremely ad hoc fashion, the transformation is generated here by the formalism. With an eye towards building on these foundations a broader formalism for higher clustering phenomena, the importance to us of this particular aspect should be clear.

More specifically, what we actually show is that, just as the standard coherent states may be viewed as eigenstates of the original single-boson destruction operator $a$, so our generalised $S U(1,1)$ coherent states may be viewed as eigenstates of a new single-boson destruction operator $b$, which is itself generated from the operators $a$ and $a^{\dagger}$ by the usual Bogoliubov canonical transformation. In other words, we demonstrate that the generalised coherent states appropriate to paired bosons that we construct may also be viewed as standard coherent states of the Bogoliubov quasiparticles.

From the discussion and motivation above, it is clear that a class of physical systems to which our results may be applied is those described by Hamiltonians at least approximately bilinear in the underlying boson fields. Apart from such obvious examples as superfluidity and the parametric excitation of a quantum oscillator, there are perhaps less obvious applications in both quantum optics and relativistic field theory and general relativity.

Within quantum optics and quantum electronics, similar states to those that we describe in $\$ 2$ have recently been described by several authors (Yuen 1976, Caves 1982, Walls 1983). Just as the standard coherent states were introduced into quantum optics by Glauber (1963) as one-photon coherent states appropriate to the radiation field from a conventional single-photon laser, so our generalised $\mathrm{SU}(1,1)$ paired coherent states may be viewed as two-photon coherent states in connection with the possibility of a two-photon laser. In this context our generalised coherent states have become known as 'squeezed' states for reasons that we discuss when we take this point up again in our concluding remarks set out in $\$ 4$.

As a concrete example of how our results may be applied we discuss in $\S 3$ an example drawn from relativity and quantum field theory, namely the relative nature of the vacuum (and other) states for an inertial observer and for a uniformly accelerated 
observer. From a mathematical point of view the fundamental operators $U_{2}(\sigma, \lambda)$, defined in $\S 2$, which generate the particular representation of the group $\operatorname{SU}(1,1)$ of relevance to us here, provide a unitary isomorphism of a Hilbert space onto itself by mapping each ket $|s\rangle$ of the space into another ket $|s ; \sigma \lambda\rangle \equiv U_{2}(\sigma, \lambda)|s\rangle$ belonging to the space. We explain in $\S 3$ that there is an interesting physical interpretation of this mapping in the relativistic case of Minkowski space. If the operators $a$ and $a^{\dagger}$ are now associated with quanta appropriate, say, to solutions of the massless Klein-Gordon equation in the Minkowski metric appropriate to an inertial observer, then it turns out that the operators $b$ and $b^{+}$are associated with the corresponding solutions in the so-called Rindler metric appropriate to an observer undergoing uniform acceleration. Some previously known results for this rather important example (which has a close bearing on the phenomenon of Hawking radiation from a black hole) can then be rather simply demonstrated and extended, using our general results.

More generally we believe that the generalised paired coherent states that we introduce in $\$ 2$, their properties and the results that we discuss there will be very useful for practical calculations in the many other fundamental problems in quantum field theory and many-body theory where the Bogoliubov transformation continues to play an important role (e.g. Hsue et al 1985). After the discussion in $\S 3$ of the particular application mentioned above, we conclude in $\$ 4$ with some remarks concerning possible extensions and generalisations of this work.

\section{The generalised coherent states}

We begin our discussion by considering the unitary operators

$$
\exp \left(A a^{\dagger}-A^{*} a\right) \exp (\mathrm{i} \phi) \quad A \in \mathbb{C} \quad \phi \in \mathbb{R}
$$

which form the so-called Weyl (or Heisenberg-Weyl) group, where $a$ and $a^{\dagger}$ are the usual boson destruction and creation operators. Together with the identity operator $I$ they satisfy the Heisenberg commutation relations

$$
\left[a, a^{\dagger}\right]=I
$$

and so generate a Lie algebra-the so-called Weyl (or Heisenberg-Weyl) algebra. The phase factor $\exp (\mathrm{i} \phi)$ plays no further role in our arguments and will henceforth be omitted. Ordinary (or standard) coherent states are defined as usual by

$$
|A\rangle \equiv U_{1}(A)|0\rangle \quad U_{1}(A) \equiv \exp \left(A a^{\dagger}-A^{*} a\right)
$$

where $|0\rangle$ is the vacuum, $a|0\rangle=0$. In terms of the states $|n\rangle$ of definite boson number

$$
a^{\dagger} a|n\rangle=n|n\rangle \quad|n\rangle=(n !)^{-1 / 2}\left(a^{\dagger}\right)^{n}|0\rangle
$$

the standard coherent state has the form

$$
|A\rangle=\exp \left(-\frac{1}{2}|A|^{2}\right) \sum_{n=0}^{\infty}(n !)^{-1 / 2} A^{n}|n\rangle
$$

and is easily seen to be an eigenstate of the destruction operator

$$
a|A\rangle=A|A\rangle \text {. }
$$


We next consider a representation of the group $\mathrm{SU}(1,1)$ realised with the unitary operators

$$
U_{2}(\rho, \theta, \lambda) \equiv \exp \left(-\frac{1}{4} \rho \mathrm{e}^{-\mathrm{i} \theta} a^{\dagger 2}+\frac{1}{4} \rho \mathrm{e}^{\mathrm{i} \theta} a^{2}\right) \exp \left(\mathrm{i} \lambda a^{\dagger} a\right) \quad \rho, \theta, \lambda \in \mathbb{R} \quad U_{2}^{\dagger} U_{2}=I .
$$

The three operators $K_{+}, K_{-}$and $K_{0}$ defined as

$$
K_{+} \equiv \frac{1}{2} a^{+2} \quad K_{-} \equiv \frac{1}{2} a^{2} \quad K_{0} \equiv \frac{1}{2} a^{\dagger} a+\frac{1}{4}
$$

satisfy the Lie algebra of $\mathrm{SU}(1,1)$, namely

$$
\left[K_{0}, K_{ \pm}\right]= \pm K_{ \pm} \quad\left[K_{-}, K_{+}\right]=2 K_{0} .
$$

In terms of the operator $U_{2}$ of equation (6) we finally introduce the states $|A ; \rho \theta \lambda\rangle$ defined as

$$
|A ; \rho \theta \lambda\rangle \equiv U_{2}(\rho, \theta, \lambda)|A\rangle=U_{2}(\rho, \theta, \lambda) U_{1}(A)|0\rangle
$$

which are the prime objects of study in this paper. We point out immediately that the states $|A\rangle$, given in equation (4), are eigenstates of the operator $K_{\text {- }}$

$$
K_{-}|A\rangle=\frac{1}{2} A^{2}|A\rangle
$$

but not of the operator $K_{0}$. We stress this point because in the literature the $\operatorname{SU}(1,1)$ coherent states are usually generated by letting $U_{2}$ or a similar operator act on an eigenstate of $K_{0}$.

Next we note the important relation

$$
\exp \left(-\frac{1}{2} \rho \mathrm{e}^{-\mathrm{i} \theta} K_{+}+\frac{1}{2} \rho \mathrm{e}^{\mathrm{i} \theta} K_{-}\right)=\mathrm{e}^{\sigma K_{+}} \mathrm{e}^{\tau K_{0}} \mathrm{e}^{-\sigma^{*} K_{-}}
$$

where

$$
\sigma \equiv-\mathrm{e}^{-\mathrm{i} \theta} \tanh \left(\frac{1}{2} \rho\right) \quad \tau \equiv \ln \left(1-|\sigma|^{2}\right)
$$

which has been given by Perelomov (1977). Equation (11) is valid for any operators $K_{+}, K_{-}$and $K_{0}$ which satisfy the algebra of equation (8), not just the particular representation of it given in equation (7). (We note that it is simplest, and sufficient, to prove equation (11) for the representation $K_{0} \rightarrow \frac{1}{2} \sigma_{3}, K_{ \pm} \rightarrow \frac{1}{2} \mathrm{i}\left(\sigma_{1} \pm \mathrm{i} \sigma_{2}\right)$ in terms of the usual Pauli spin matrices $\sigma_{i}, i=1,2,3$.) Using the result of equations (11) and (12), we may write the operator $U_{2}(\rho, \theta, \lambda)$ of equation (6) in the equivalent form

$$
U_{2}(\sigma, \lambda)=\exp \left(\frac{1}{2} \sigma a^{\dagger 2}\right)\left(1-|\sigma|^{2}\right)^{a^{\dagger} a / 2+1 / 4} \exp \left(-\frac{1}{2} \sigma^{*} a^{2}\right) \exp \left(\mathrm{i} \lambda a^{+} a\right)
$$

where $\lambda$ is real and $\sigma$, given by equation (12), is complex and with modulus $|\sigma|<1$. Equation (13) is particularly useful in simplifying the derivation of later results.

For example, we can easily prove the very important relations

$$
\begin{aligned}
& U_{2}(\sigma, \lambda) a U_{2}^{\dagger}(\sigma, \lambda)=\mathrm{e}^{-\mathrm{i} \lambda}\left(1-|\sigma|^{2}\right)^{-1 / 2}\left(a-\sigma a^{\dagger}\right) \equiv b \\
& U_{2}(\sigma, \lambda) a^{\dagger} U_{2}^{\dagger}(\sigma, \lambda)=\mathrm{e}^{\mathrm{i} \lambda}\left(1-|\sigma|^{2}\right)^{-1 / 2}\left(a^{\dagger}-\sigma^{*} a\right) \equiv b^{\dagger}
\end{aligned}
$$

either by making use of equation (13) and the unitarity of $U_{2}$, or directly from equation (6). We readily see from equation (14) that the operators $b$ and $b^{+}$again obey the boson commutation relations

$$
\left[b, b^{\dagger}\right]=I
$$

and that the transformation from the operators $a$ and $a^{\dagger}$ to the operators $b$ and $b^{+}$is just the usual Bogoliubov transformation. From equation (14) and the fact that $U_{2}$ is unitary we can trivially prove for any function $f\left(a, a^{+}\right)$the relation

$$
U_{2} f\left(a, a^{\dagger}\right) U_{2}^{\dagger}=f\left(b, b^{\dagger}\right) \leftrightarrow U_{2} f\left(a, a^{+}\right)=f\left(b, b^{\dagger}\right) U_{2} .
$$


By making use of equation (13) and the trivial relation

$$
\exp \left(\phi a^{\dagger} a\right)|A\rangle=\exp \left[\frac{1}{2}|A|^{2}\left(\left|\mathrm{e}^{\phi}\right|^{2}-1\right)\right]\left|A \mathrm{e}^{\phi}\right\rangle
$$

which is readily proved from equations (3) and (4), we can now write the states of equation (9) in the equivalent form

$$
\begin{aligned}
&|A ; \sigma \lambda\rangle \equiv U_{2}(\sigma, \lambda)|A\rangle \\
&=U_{2}(\sigma, \lambda) U_{1}(A)|0\rangle \\
&=\left(1-|\sigma|^{2}\right)^{1 / 4} \exp \left(-\frac{1}{2} \sigma^{*} A^{2} \mathrm{e}^{2 i \lambda}-\frac{1}{2}|\sigma A|^{2}\right) \\
& \times \exp \left(\frac{1}{2} \sigma a^{\dagger 2}\right)\left|A \mathrm{e}^{\mathrm{i} \lambda}\left(1-|\sigma|^{2}\right)^{1 / 2}\right\rangle .
\end{aligned}
$$

In the special case $\lambda=0=\sigma$, we simply get the standard coherent states, $|A ; 00\rangle=|A\rangle$, defined in equation (2). Equation (16) immediately implies that $U_{2} a=b U_{2}$, and hence that the states $|A ; \sigma \lambda\rangle$ are eigenstates of the destruction operator $b$,

$$
\begin{aligned}
b|A ; \sigma \lambda\rangle & =b U_{2}(\sigma, \lambda)|A\rangle \\
& =U_{2}(\sigma, \lambda) a|A\rangle \\
& =A|A ; \sigma \lambda\rangle
\end{aligned}
$$

by making use of equation (5). The special case $A=0$ is of particular interest, since equation (19) implies the relation

$$
b|0 ; \sigma\rangle=0
$$

where we have written $|0 ; \sigma \lambda\rangle \equiv|0 ; \sigma\rangle$, as equation (18) shows that this state is independent of $\lambda$. Just as the state $|0\rangle$ was defined by the relation $a|0\rangle=0$ to be the ground state with respect to the operators $a$ and $a^{+}$(i.e. the vacuum for $a$-type bosons), so the state $|0 ; \sigma\rangle \equiv U_{2}(\sigma, \lambda)|0\rangle$ obeys equation (20) and is therefore the vacuum for $b$-type bosons. By making further use of equation (16) we get the relation

$$
U_{2}(\sigma, \lambda) \exp \left(A a^{\dagger}-A^{*} a\right)=\exp \left(A b^{+}-A^{*} b\right) U_{2}(\sigma, \lambda)
$$

and hence from equations (2), (9) and (21):

$$
|A ; \sigma \lambda\rangle=\exp \left(A b^{\dagger}-A^{*} b\right)|0 ; \sigma\rangle .
$$

Thus we see very clearly that the states $|A ; \sigma \lambda\rangle$ may be viewed as ordinary coherent states with respect to the operators $b$ and $b^{\dagger}$, i.e. they are the standard coherent states of the Bogoliubov quasiparticles (or $b$-type bosons). For fixed values of $\sigma$ and $\lambda$, they therefore obey the well known properties of ordinary coherent states, e.g.,

$$
\begin{aligned}
& \langle D ; \sigma \lambda \mid A ; \sigma \lambda\rangle=\exp \left(D^{*} A-\frac{1}{2}|D|^{2}-\frac{1}{2}|A|^{2}\right) \\
& |\langle D ; \sigma \lambda \mid A ; \sigma \lambda\rangle|^{2}=\exp \left(-|D-A|^{2}\right) \\
& \int \frac{\mathrm{d}^{2} A}{\pi}|A ; \sigma \lambda\rangle\langle A ; \sigma \lambda|=I
\end{aligned}
$$

where

$$
\begin{aligned}
& \mathrm{d}^{2} A \equiv \mathrm{d}(\operatorname{Re} A) \mathrm{d}(\operatorname{Im} A) \\
& |A ; \sigma \lambda\rangle=\exp \left(-\frac{1}{2}|A|^{2}\right) \sum_{n=0}^{\infty}(n !)^{-1 / 2} A^{n}|n ; \sigma \lambda\rangle \\
& |n ; \sigma \lambda\rangle=U_{2}(\sigma, \lambda)|n\rangle
\end{aligned}
$$


where the states $|n ; \sigma \lambda\rangle$ are eigenstates of the number operator $b^{\dagger} b$ for $b$-type bosons:

$$
b^{\dagger} b|n ; \sigma \lambda\rangle=n|n ; \sigma \lambda\rangle \quad|n ; \sigma \lambda\rangle=(n !)^{-1 / 2}\left(b^{\dagger}\right)^{n}|0 ; \sigma\rangle .
$$

In terms of the eigenstates $|m\rangle$ of the number operator $a^{\dagger} a$, we now wish to calculate the overlaps $\langle m \mid n ; \sigma \lambda\rangle$. We start by using equation (18) with $A=0$ to get

$$
\langle 0 \mid 0 ; \sigma\rangle=\left(1-|\sigma|^{2}\right)^{1 / 4} \text {. }
$$

We next use the relation $\langle m|b| 0 ; \sigma\rangle=0$ obtained from equation (20), together with the definition of the operator $b$ from equation (14), to obtain the recursion relation

$$
\langle m+1 \mid 0 ; \sigma\rangle=\sigma\left(\frac{m}{m+1}\right)^{1 / 2}\langle m-1 \mid 0 ; \sigma\rangle .
$$

Finally, we use the relation

$$
\langle m \mid n+1 ; \sigma \lambda\rangle=(n+1)^{-1 / 2}\left\langle m\left|b^{\dagger}\right| n ; \sigma \lambda\right\rangle
$$

which follows trivially from equation (26), together with the definition of $b^{\dagger}$ from equation (14), to obtain the recursion relation

$$
\begin{aligned}
\langle m \mid n+1 ; \sigma \lambda\rangle & =\mathrm{e}^{\mathrm{i} \lambda}\left(1-|\sigma|^{2}\right)^{-1 / 2} \\
& \times\left[\left(\frac{m}{n+1}\right)^{1 / 2}\langle m-1 \mid n ; \sigma \lambda\rangle-\sigma^{*}\left(\frac{m+1}{n+1}\right)^{1 / 2}\langle m+1 \mid n ; \sigma \lambda\rangle\right] .
\end{aligned}
$$

It is clear that all of the overlap integrals $\langle m \mid n ; \sigma \lambda\rangle$ can be recursively obtained from equations (27), (28) and (30).

We may also compare the ordinary coherent states $|A\rangle$ (with $\sigma=0=\lambda$ ) with our generalised coherent states $|B ; \sigma \lambda\rangle$ in the following way. Use of equations (18) and (5), together with the analogous relation to equation (23) for the overlap integrals for ordinary coherent states, gives the relation

$$
\begin{aligned}
\langle A \mid B ; \sigma \lambda\rangle= & \left(1-|\sigma|^{2}\right)^{1 / 4} \\
& \times \exp \left[-\frac{1}{2} \sigma^{*} B^{2} \mathrm{e}^{2 \mathrm{i} \lambda}+\frac{1}{2} \sigma A^{* 2}+A^{*} B \mathrm{e}^{\mathrm{i} \lambda}\left(1-|\sigma|^{2}\right)^{1 / 2}-\frac{1}{2}|A|^{2}-\frac{1}{2}|B|^{2}\right] .
\end{aligned}
$$

It is clear that the states $|A\rangle$ and $|B ; \sigma \lambda\rangle$ are not orthogonal in general, but that they become more nearly so as $|\sigma|$ approaches unity. Use of the completeness relation for the ordinary coherent states $|A\rangle$, analogous to equation (24), yields the expansion

$$
|B ; \sigma \lambda\rangle=\int \frac{\mathrm{d}^{2} A}{\pi}\langle A \mid B ; \sigma \lambda\rangle|A\rangle
$$

for the generalised coherent states in terms of the standard coherent states, and where the overlap integrals $\langle A \mid B ; \sigma \lambda\rangle$ are given explicitly in equation (31). A particularly interesting special case of equations (31) and (32) is the resolution of the vacuum for $b$-type bosons:

$$
|0 ; \sigma\rangle=\left(1-|\sigma|^{2}\right)^{1 / 4} \int \frac{\mathrm{d}^{2} A}{\pi} \exp \left(\frac{1}{2} \sigma A^{* 2}-\frac{1}{2}|A|^{2}\right)|A\rangle .
$$

Finally, explicit use of the definitions from equation (14) of the operators $b$ and $b^{\dagger}$ in terms of the operators $a$ and $a^{+}$gives the very important relation

$$
\left\langle 0\left|b^{+} b\right| 0\right\rangle=\left(|\sigma|^{-2}-1\right)^{-1}
$$

for the expectation value of the number of $b$-type bosons in the vacuum state for $a$-type bosons. 


\section{Application: an example from relativity}

We turn now to an illustration of the use of these generalised coherent states and their associated Bogoliubov transformation, drawn from the fields of relativity and quantum field theory. In the relativistic case of Minkowski flat space it has been known for some time that a Bogoliubov transformation analogous to equation (14) may be associated with a uniformly accelerated observer (Davies 1975, 1978, Unruh 1976, Candelas and Raine 1976, Sciama et al 1981). Our results can in this case be given a very interesting physical interpretation.

We first review the physical problem that we have in mind. Let us consider the usual Minkowski coordinates $(t, x, y, z)$, in units with $c=1$, for the inertial (or Minkowski) observer who sees the spacetime line element

$$
\mathrm{d} s^{2}=-\mathrm{d} t^{2}+\mathrm{d} x^{2}+\mathrm{d} y^{2}+\mathrm{d} z^{2}
$$

and also the Rindler coordinates $(\tau, \xi, y, z)$

$$
x=\xi \cosh \tau \quad t=\xi \sinh \tau
$$

for the so-called Rindler observer undergoing uniform acceleration, in terms of which the spacetime line element (35) becomes

$$
\mathrm{d} s^{2}=-\xi^{2} \mathrm{~d} \tau^{2}+\mathrm{d} \xi^{2}+\mathrm{d} y^{2}+\mathrm{d} z^{2}
$$

In this case, lines of constant $\xi$ thus correspond to worldlines of (Rindler) observers undergoing uniform proper acceleration $\xi^{-1}$ and whose proper time is $\xi \tau$.

Our aim is now to quantise the massless Klein-Gordon equation for a scalar field $\phi$ in the Rindler coordinates. A complete set of solutions to this classical equation in the so-called Rindler wedge $(|x|>|t|)$, is readily shown to be given by

$$
u_{\omega k}(\tau \xi y z)=N_{\omega k} \exp \left(-\mathrm{i} \omega \tau+\mathrm{i} k_{2} y+\mathrm{i} k_{3} z\right) K_{\mathrm{i} \omega}(k \xi) \quad \boldsymbol{k} \equiv\left(k_{2}, k_{3}\right) \quad k=|\boldsymbol{k}|
$$

where $K_{\mathrm{i} \omega}(x)$ is a Bessel function of the third kind and $N_{\omega k}$ is a suitable normalisation constant. We note that in the right Rindler region $(x>|t|)$ the solutions $u_{\omega k}$ represent the positive frequency Rindler modes and $u_{\omega k}^{*}$ the corresponding negative frequency modes, where the terms positive and negative frequency are determined with respect to the operator $\mathrm{i} \partial / \partial \tau$. Conversely, it is not difficult to see that in the left Rindler region $(x<-|t|)$, the solutions $u_{\omega k}$ represent the negative frequency modes and the $u_{\omega k}^{*}$ the positive frequency modes. As usual we now define $u_{\omega k}^{(+)}$to be that solution of the wave equation which vanishes in the left Rindler wedge and $\boldsymbol{u}_{\boldsymbol{\omega}}^{(-)}$to be the corresponding solution which vanishes in the right Rindler wedge, and where in each case the solution equals $u_{\omega k}$ in the opposing Rindler wedge. For the uniformly accelerated observer, quantisation of the scalar field is now based on the canonical mode expansion:

$$
\phi(\tau \xi y z)=\int_{0}^{\infty} \mathrm{d} \omega \int \frac{\mathrm{d}^{2} k}{(2 \pi)^{2}}\left(u_{\omega k}^{(+)} b_{\omega k}^{(+)}+u_{\omega k}^{(-)} b_{\omega k}^{(-)}+\mathrm{HC}\right)
$$

where $\mathrm{HC}$ indicates the Hermitian conjugate, and the operators $b_{\omega k}^{( \pm)}$obey the usual boson canonical commutation relations

$$
\left[b_{\omega k}^{(+)}, b_{\omega^{\prime} k^{\prime}}^{(+)}\right]=\delta\left(\omega-\omega^{\prime}\right) \delta\left(k-k^{\prime}\right)=\left[b_{\omega k}^{(-)}, b_{\omega^{\prime} k^{\prime}}^{(-)^{\dagger}}\right]
$$

with all other commutators vanishing.

Now the solutions $u_{\omega \boldsymbol{k}}^{(+)}$and $\boldsymbol{u}_{\omega \boldsymbol{k}}^{(-)}$are analytic everywhere except for the horizons $(x \pm t=0)$, and the same is therefore true for general linear combinations of them. It 
can however be verified that the particular combinations

$$
v_{\omega k}^{( \pm)} \equiv[1-\exp (-2 \pi \omega)]^{-1 / 2}\left[u_{\omega k}^{( \pm)}+\exp (-\pi \omega) u_{\omega k}^{(\mp)}\right]
$$

are also analytic on these horizons. In this case they may therefore be used for a Minkowski decomposition of the field $\phi$ for the inertial observer. One can also prove that the solutions $v_{\omega k}^{(+)}$and $v_{\omega k}^{(-)}$are the corresponding positive and negative frequency modes for the Minkowski observer, i.e. with respect to the operator i $\partial / \partial t$. The corresponding Minkowski quantisation of the scalar field $\phi$, with the normalisation factors of equation (41), may then be given as

$$
\phi(t x y z)=\int_{0}^{\infty} \mathrm{d} \omega \int \frac{\mathrm{d}^{2} k}{(2 \pi)^{2}}\left(v_{\omega k}^{(+)} a_{\omega k}^{(+)}+v_{\omega k}^{(-) *} a_{\omega k}^{(-)}+\mathrm{HC}\right)
$$

and the operators $a_{\omega k}^{( \pm)}$obey analogous commutation relations to the operators $b_{\omega k}^{( \pm)}$.

A comparison of equations (39) and (42), together with the defining relations (41), easily leads to the relation

$$
b_{\omega k}^{( \pm)}=\left(1-\mathrm{e}^{-2 \pi \omega}\right)^{-1 / 2}\left(a_{\omega k}^{( \pm)}+\mathrm{e}^{-\pi \omega} a_{\omega k}^{(\neq) \dagger}\right)
$$

between the boson operators in the two decompositions. If we then define new operators

$$
a_{\omega k} \equiv \mathrm{e}^{\mathrm{i} \gamma}\left(a_{\omega k}^{(+)}+a_{\omega k}^{(-)}\right) \quad b_{\omega k} \equiv \mathrm{e}^{\mathrm{i} \gamma}\left(b_{\omega k}^{(+)}+b_{\omega k}^{(-)}\right)
$$

for arbitrary real $\gamma$, we find the fundamental Bogoliubov transformation

$$
b_{\omega k}=\left(1-\mathrm{e}^{-2 \pi \omega}\right)^{-1 / 2}\left[a_{\omega k}+\exp (-\pi \omega+2 \mathrm{i} \gamma) a_{\omega k}^{\dagger}\right] .
$$

A comparison of equations (45) and (14) then reveals that the present example is simply related to our general discussion with the identification

$$
\sigma=-\exp (-\pi \omega+2 \mathrm{i} \gamma) \text {. }
$$

Hence, all of our previous results may be applied immediately.

In particular the states $|0\rangle$ and $|0 ; \sigma\rangle$ now play the role of the vacua for our massless scalar meson field as observed by the (inertial) Minkowski and (uniformly accelerated) Rindler observers, respectively. More generally the operator $U_{2}(\sigma, \lambda)$ defines a unitary isomorphism of the Hilbert space $H$ onto itself by mapping each ket $|s\rangle$ into the ket $|s ; \sigma \lambda\rangle \equiv U_{2}(\sigma, \lambda)|s\rangle$. Corresponding to each operator $\Theta=f\left(a, a^{\dagger}\right)$, we map the operator $\Theta_{\sigma, \lambda} \equiv U_{2}(\sigma, \lambda) f\left(a, a^{+}\right) U_{2}^{\dagger}(\sigma, \lambda)=f\left(b, b^{+}\right)$, as in equation (16). If the operator $\Theta$ is attributed to the inertial observer, then $\Theta_{\sigma, \lambda}$ is the corresponding operator for the accelerated observer. It is trivial to prove that if $|\theta\rangle$ is an eigenstate of the inertial observer's operator $\Theta$, then the corresponding eigenstate of the accelerated observer's operator $\Theta_{\sigma, \lambda}$ is $|\theta ; \sigma \lambda\rangle \equiv U_{2}(\sigma, \lambda)|\theta\rangle$. We present here two examples of these results.

In the first place, it is convenient for many purposes to define the Hermitian operators

$$
\hat{x} \equiv 2^{-1 / 2} \mathrm{i}\left(a-a^{\dagger}\right) \quad \hat{p} \equiv 2^{-1 / 2}\left(a+a^{\dagger}\right)
$$

which, from equation (1), obey the commutation relation $[\hat{x}, \hat{p}]=\mathrm{i} I$, and hence (in suitable units) play the role of position and linear momentum operators respectively. Suppose now that $\left|x_{0}\right\rangle$ is an eigenstate of the inertial observer's position operator $\hat{x}$. Then, from our general result above, $\left|x_{0} ; \sigma \lambda\right\rangle \equiv U_{2}(\sigma, \lambda)\left|x_{0}\right\rangle$ is the corresponding eigenstate of the accelerated observer's position operator $\hat{x}_{\sigma, \lambda}$ :

$$
\hat{x}_{\sigma, \lambda}=U_{2}(\sigma, \lambda) \hat{x} U_{2}^{\dagger}(\sigma, \lambda)=2^{-1 / 2} \mathbf{i}\left(b-b^{\dagger}\right)
$$


with the same eigenvalue $x_{0}$ :

$$
\hat{x}\left|x_{0}\right\rangle=x_{0}\left|x_{0}\right\rangle \quad \hat{x}_{\sigma, \lambda}\left|x_{0} ; \sigma \lambda\right\rangle=x_{0}\left|x_{0} ; \sigma \lambda\right\rangle .
$$

We can now calculate these eigenstates in, say, the $x$ representation, $\left\langle x \mid x_{0} ; \sigma \lambda\right\rangle$, as follows. We first use equations (14) and (47) to rewrite relation (48) in the form

$$
\begin{gathered}
\hat{x}_{\sigma, \lambda}=\frac{1}{2}\left(1-|\sigma|^{2}\right)^{-1 / 2}\left\{\left[(1+\sigma) \mathrm{e}^{-\mathrm{i} \lambda}+\left(1+\sigma^{*}\right) \mathrm{e}^{\mathrm{i} \lambda}\right] \hat{x}\right. \\
\left.+\mathrm{i}\left[(1-\sigma) \mathrm{e}^{-\mathrm{i} \lambda}-\left(1-\sigma^{*}\right) \mathrm{e}^{\mathrm{i} \lambda}\right] \hat{p}\right\} .
\end{gathered}
$$

Inserting equation (50) into equation (49) and making use of the usual representations of the operators $\hat{x}$ and $\hat{p}$,

$$
\langle x| \hat{x}=x\langle x| \quad\langle x| \hat{p}=-\mathrm{i}(\mathrm{d} / \mathrm{d} x)\langle x|
$$

we then readily find the result

$$
\left\langle x \mid x_{0} ; \sigma \lambda\right\rangle=(\text { constant }) \exp \left(\frac{2\left(1-|\sigma|^{2}\right)^{1 / 2} x_{0} x-\frac{1}{2}\left[(1+\sigma) \mathrm{e}^{-\mathrm{i} \lambda}+\left(1+\sigma^{*}\right) \mathrm{e}^{\mathrm{i} \lambda}\right] x^{2}}{(1-\sigma) \mathrm{e}^{-\mathrm{i} \lambda}-\left(1-\sigma^{*}\right) \mathrm{e}^{\mathrm{i} \lambda}}\right) .
$$

It is of special interest to note that for the particular case when $\sigma$ is real $(=u$, say) and $\lambda=0$, the accelerated observer's eigenfunction approaches a Dirac delta function, exactly as for the inertial observer, but situated not at $x_{0}$ (as for the inertial observer) but rather at the point $[(1-u) /(1+u)]^{1 / 2} x_{0}$.

As a second example we consider precisely the coherent states discussed in $\S 2$. In this case, if $|A\rangle$ is a coherent state for the inertial observer satisfying equation (5), then $|A ; \sigma \lambda\rangle$ is the corresponding coherent state for the accelerated observer satisfying equation (19). We conclude this section with a particularly important result. A direct combination of equations (34) and (46) then gives the most interesting observation that the Minkowski vacuum $|0\rangle$ appears to the uniformly accelerated observer, for whom the meson number operator is $b_{\omega k}^{\dagger} b_{\omega k}$, as a black-body radiator with Planckian distribution

$$
\left\langle 0\left|b_{\omega k}^{\dagger} b_{\omega k}\right| 0\right\rangle=\left(\mathrm{e}^{2 \pi \omega}-1\right)^{-1}
$$

corresponding to a 'temperature' $\left(2 \pi k_{\mathrm{B}}\right)^{-1}$ in our units, where $k_{\mathrm{B}}$ is the Boltzmann constant. In order to express equation (53) in ordinary units, we realise that along lines of constant $\xi$ an interval of proper time is given by $\xi \mathrm{d} \tau$. Thus, an observer with Rindler coordinates $(\tau, \xi, y, z)$ interprets a wave with time dependence $\exp (i \omega \tau)$ as having an angular frequency $\omega / \xi$. Hence, from equation (53) we see that to such a uniformly accelerated observer the Minkowski vacuum appears to have a temperature

$$
T=\frac{\hbar}{2 \pi k_{\mathrm{B}} c \xi}
$$

which is directly proportional to the proper acceleration $\xi^{-1}$. Davies (1975) seems to have been the first to realise that the Rindler vacuum contains a thermal distribution of quanta relative to the Minkowski vacuum. Sciama et al (1981) have stressed how an observer undergoing uniform acceleration, and hence finding his spacetime manifold bounded by an event horizon, can be thereby profitably viewed as constituting a 'model' black hole. They have further shown the deep connection of the result that we have just demonstrated with the thermal properties of real black holes when quantum effects 
are taken into account, and in particular with the obviously reminiscent phenomenon of Hawking radiation.

\section{Conclusions}

In this paper we have investigated the generalised coherent states $|A ; \sigma \lambda\rangle \equiv U_{2}(\sigma, \lambda)|A\rangle$, obtained from the standard coherent states $|A\rangle \equiv U_{1}(A)|0\rangle$ by letting the operator $U_{2}(\sigma, \lambda)$ defined in equation (13) act on them, just as the standard coherent states are themselves built by letting the displacement operator $U_{1}(A)$ of equation (2) act on the vacuum. The 'coherence' properties of the standard coherent states $|A\rangle$ are already contained in the exponentiation of the one-body operator contained in equation (2), where by one-body operator in this context we mean one containing terms linear in the operators $a$ and $a^{\dagger}$. Similarly, the operator $U_{2}(\sigma, \lambda)$ of equation (13) is an exponential of an operator bilinear in the operators $a$ and $a^{\dagger}$ and may therefore be expected a priori to generate a coherent paired state. We have shown how this generalised coherent state can itself be alternatively viewed as a standard coherent state with respect to new boson (quasiparticle) operators that stand in relation to the original boson (particle) operators by a Bogoliubov transformation. We have further shown how these generalised coherent states may be associated with the Lie group $\mathrm{SU}(1,1)$ and we have examined their properties in some detail.

One knows that the standard coherent states have a number of interesting properties that make them particularly suitable for physical applications. Probably foremost among these is that an expansion of an appropriate quantum field in such states makes the taking of the classical limit particularly easy and transparent. This property is itself connected with the fact that the standard coherent states minimise the Heisenberg (position-momentum) uncertainty relation (i.e. for these states the inequality is satisfied as an equality), and for this reason it is not surprising that they are the quantal states that most closely correspond to classical behaviour.

We note parenthetically that coherent states are often initially approached from their 'minimum uncertainty' property, as for example in quantum optics. We have already remarked in $\S 1$ on the possible use in quantum optics of our generalised $\mathrm{SU}(1,1)$ paired coherent states as two-photon coherent states in connection with the possibility of a two-photon laser, where they have been called 'squeezed states' for reasons which we now explain. In terms of a quantum harmonic oscillator with 'mass' and 'circular frequency' parameters $m$ and $\omega$ respectively, one can as usual introduce canonically conjugate 'position' and 'momentum' operators $\hat{x}$ and $\hat{p}$ respectively, by the relation $a \equiv(2 m \hbar \omega)^{-1 / 2} \hat{p}-\mathrm{i}(m \omega / 2 \hbar)^{1 / 2} \hat{x}$. In this case it is not difficult to show that the standard coherent states $|A\rangle$ minimise the position-momentum uncertainty relation, $\Delta p \Delta x=\frac{1}{2} \hbar$, with a common ratio of uncertainties, $\Delta p / \Delta x=m \omega$, for all complex values of $A$. Since our generalised coherent states $|A ; \sigma \lambda\rangle$ may, on the one hand, be viewed as ordinary coherent states with respect to quasiparticle operators $b$ and $b^{+}$, it comes as no surprise that they also satisfy the minimum uncertainty relation $\Delta p \Delta x=\frac{1}{2} \hbar$. However, one can also show relatively easily that the ratio $\Delta p / \Delta x$ for the generalised coherent states is now 'squeezed' in the sense that it acquires an extra factor $(1+\sigma) /(1-$ $\sigma)$ relative to the value quoted above for the standard coherent states.

In view of their mode of construction, we expect that our generalised coherent states will be of particular use in any quantum field theory that has an underlying dynamical symmetry of the group $S U(1,1)$, or to which the Bogoliubov transformation 
may profitably be applied. With regard to the group theoretic aspect, one should also note that $\mathrm{SU}(1,1)$ is not the only appropriate group here. For example, the threedimensional Lorentz group $\mathrm{SO}(2,1)$ which is the group of rotations in three-dimensional Minkowski space, is locally isomorphic to $\operatorname{SU}(1,1)$, and indeed also to both the group $\operatorname{SL}(2, R)$ of real second-order matrices with unit determinant and the symplectic group $\operatorname{Sp}(2, R)$.

Returning to our original idea of building coherent paired states, it is possible to imagine several generalisations of the current approach. In the first place one may imagine generalising from a single boson (or canonical quantum mode) to the case of several distinct bosons (or modes). In the case of $n$ bosons or modes, the various bilinear products of operators $a_{i}^{\dagger} a_{j}^{\dagger}, a_{i} a_{j}$ and $a_{i}^{\dagger} a_{j}, i, j=1, \ldots, n$, now form a basis for a realisation of the higher symplectic algebra $\operatorname{Sp}(2 n, R)$. As before, one can similarly construct a unitary realisation of this group by exponentiating the skew-adjoint operators in this algebra. It should then be possible to extend in an obvious manner the treatment given here to the case of $n$ distinct bosons or modes.

A potentially much more important generalisation is concerned with our original motivation, namely to investigate higher clustering correlations in a similar fashion. Thus, for correlated clusters of $m$ identical bosons (within a many-boson system), one can imagine examining the underlying group structure of the various products of order $m$ in the operators $a$ and $a^{+}$, and in this way constructing appropriate coherent states for these $m$ clusters. We hope in this way to be able to generalise the Bogoliubov transformation appropriate to (a particular type of) pairing, to transformations suitable for describing higher clustering phenomena and presumably also the possible new condensed phases that would be associated with them. Finally, it would also be of interest to extend any results obtained for bosonic systems to systems of many fermions.

By way of final conclusions we note that the 'squeezed states' analogous to our generalised paired coherent states have attracted much recent work and excitement in quantum optics. By analogy, it is our belief and hope both that there are many other similar problems to which the present results might usefully be applied, and also that their possible generalisations outlined above will find immediate and potentially far-reaching applicability in both quantum optics and condensed matter theory.

\section{Acknowledgments}

We gratefully acknowledge support for this work in the form of a research grant from the Science and Engineering Research Council of Great Britain. One of us (AV) would also like to thank $\mathrm{H}$ Kümmel for useful discussions.

\section{References}

Barut A O and Girardello L 1971 Commun. Math. Phys. 2141

Candelas P and Raine D J 1976 J. Math. Phys. 172101

Caves C M 1982 Phys. Rev. D 261817

Davies P C W 1975 J. Phys. A: Math. Gen. 8609

- 1978 Rep. Prog. Phys. 411313

Feshbach H and Tikochinsky Y 1977 Trans. NY Acad. Sci. 3844

Glauber R J 1963 Phys. Rev. 1312766

Hsue C S, Kümmel H and Ueberholz P 1985 Ruhr-Universität Bochum preprint 
Nieto M M and Simmons L M Jr 1979 Phys. Rev. D 20 1321, 1332, 1342

Perelomov A M 1972 Commun. Math. Phys. 26222

1975 Commun. Math. Phys. 44197

— 1977 Usp. Fiz. Nauk 12323 (Sov. Phys.-Usp. 20 703)

Radcliffe J M 1971 J. Phys. A: Math. Gen. 4313

Sciama D W, Candelas P and Deutsch D 1981 Adv. Phys. 30327

Unruh W G 1976 Phys. Rev. D 14870

Walls D F 1983 Nature 306141

Yuen H P 1976 Phys. Rev. A 132226 